\title{
Adenosine deaminase and adenosine kinase expression in human glioma and their correlation with glioma-associated epilepsy
}

\author{
JUN HUANG $^{1}$, YUJIAO HE ${ }^{2}$, MINGNA CHEN ${ }^{2}$, JUAN DU ${ }^{2}$, \\ GUOLIANG LI ${ }^{2}$, SHUYU LI ${ }^{2}$, WEIPING LIU ${ }^{2}$ and XIAOYAN LONG ${ }^{2}$ \\ Departments of ${ }^{1}$ Neurosurgery and ${ }^{2}$ Neurology, Xiangya Hospital of Central South University, \\ Changsha, Hunan 410008, P.R. China
}

Received November 11, 2014; Accepted August 5, 2015

DOI: $10.3892 / \mathrm{mmr} .2015 .4285$

\begin{abstract}
The aim of the present study was to investigate adenosine deaminase (ADA) and adenosine kinase (ADK) expression in human glioma and to explore its correlation with glioma-associated epilepsy. Tumor tissues $(n=45)$ and peritumoral tissues $(\mathrm{n}=14)$ were obtained from glioma patients undergoing surgery. Normal control tissues $(n=8)$ were obtained from brain trauma patients. The disease grade was determined by histological evaluation and the degree of tumor invasion was evaluated using immunofluorescence analyses. mRNA and protein expression of ADA and ADK were evaluated using reverse transcription quantitative polymerase chain reaction or western blot analysis, respectively. Based on histological evaluations, four cases were classified as Grade I gliomas, 18 cases as Grade II, 17 cases as Grade III and six cases were considered Grade IV. Increased ADA and ADK expression was observed in tumor tissues. ADA was predominantly distributed in the cytoplasm of tumor cells, whereas ADK was detected in the cytoplasm as well as in the nuclei. ADA and ADK levels were upregulated in patients with Grade II and Grade III gliomas compared to those in control subjects $(\mathrm{p}<0.05)$. In addition, tumor invasion was detected in peritumoral tissues. The number of ADA-positive or ADK-positive cells in tumor tissues was similar between glioma patients with and without epilepsy $(\mathrm{p}>0.05)$. However, ADA and ADK expression was upregulated in peritumoral tissues derived from patients with epilepsy compared to that in glioma patients without epilepsy. The results of the present study suggested that ADA and ADK are involved in glioma progression, and that increased ADA and ADK levels in peritumoral tissues may be associated with epilepsy in glioma patients.
\end{abstract}

Correspondence to: Dr Xiaoyan Long, Department of Neurology, Xiangya Hospital of Central South University, 87 Xiangya Road, Changsha, Hunan 410008, P.R. China

E-mail: longxyan@sina.com

Key words: glioma, epilepsy, adenosine deaminase, adenosine kinase, peritumoral tissue

\section{Introduction}

Epilepsy is a common clinical manifestation of primary brain tumors. The prevalence of epilepsy was reported to be $>89 \%$ among patients with low-grade gliomas. Overall, epilepsy has been estimated to develop in up to $50 \%$ of glioma patients (1-3). However, surgical resection of the tumor mass does not cure epilepsy in a significant percentage of the glioma patients. Studies have indicated a variety of factors which may be linked to the incidence of glioma-associated epilepsy, including genetic background (4), tumor location (5), glioma differentiation status (6) and alternation of the peritumoral environment [e.g., neuronal cell migration (7), enhanced cell-cell communication (8) and increased glutamate concentration (9)]. To date, the pathogenesis of glioma-associated epilepsy has largely remained elusive.

Adenosine, an endogenous regulator of the mammalian brain, has essential anti-convulsive and neuroprotective roles $(10,11)$ and adenosine levels have been demonstrated to rise during seizure activity (12). Maintenance of adenosine homeostasis depends on metabolic clearance through an evolutionarily-conserved phosphotransferase, adenosine kinase (ADK), which converts the purine ribonucleoside adenosine into 5'-adenosine-monophosphate (13). In a European study of glioma patients with epilepsy, ADK upregulation was observed in peritumoral infiltrated tissues (14). In addition, adenosine deaminase (ADA) is known to be involved in purine metabolism, which deaminates adenosine and converts it to the respective nucleoside inosine. Of note, a study using an adult zebrafish model showed that ADA levels were significantly increased during pentylenetetrazole-induced seizures (15).

To gain further insight into the potential involvement of ADA and ADK in glioma-associated epilepsy, the present study was designed to perform a comparative analysis of ADA and ADK expression in human glioma tissues, peritumoral tissues and normal brain tissues. The findings of the present study enhanced the overall understanding of the pathogenesis of glioma-associated epilepsy.

\section{Materials and methods}

Patients. From April 2012 to July 2012, a total of 45 patients receiving surgical resection of gliomas at the Department 
of Neurosurgery of XiangYa Hospital (Changsha, China) were recruited for the present study. All patients provided informed written consent. Among these patients, 30 subjects were male and 15 were female. The age range of the patients was 2-65 years, with an average age of $38.24 \pm 15.82$ years. Tumor tissues and peritumoral tissues were obtained from these patients and histologically analyzed to determine the glioma grade (Grades I-IV) according to the World Health Organization (WHO) Grading of Tumours of the Central Nervous System from 2007 (16).

Normal brain tissue samples were obtained from eight brain trauma patients undergoing nerve decompression or debridement surgery. XiangYa Hospital of Central South University (Changsha, China) granted ethical approval of the present study.

Enrolment criteria. Exclusion criteria for glioma tissues were as follows: i) Ischemic lesions, hemorrhage, vascular malformation or trauma detected by magnetic resonance imaging; ii) central nervous system (CNS) disorders other than glioma and epilepsy; and iii) any other severe disorders. Inclusion criteria of peritumoral tissues were as follows: i) Clear morphological differences from tumor tissues upon gross observation; ii) location adjacent to tumor tissues; and iii) tumor cell invasion as detected using microscopy with normal cells also observed. Control subjects were included in the present study if they fulfilled the following criteria: i) No epilepsy; ii) no other CNS disorders, iii) no other severe disorders; and iv) normal brain tissue structures confirmed during pathological examination.

Reagents. The rabbit anti-ADA polyclonal antibody was purchased from Atlas (AlbaNova University Center, Stockholm, Sweden; cat. no. HPA023884). The rabbit anti-ADK (cat. no. PA5-13860) and mouse anti-p53 (cat. no. DO-7+BP53-12) polyclonal antibodies were obtained from Pierce (Thermo Fisher Scientific, Waltham, MA, USA). Monoclonal mouse anti-Ki67 was obtained from Dako (Glostrup, Denmark; cat. no. MIB-1) and polyclonal rabbit anti-glial fibrillary acidic protein (GFAP; cat. no. ZA-0529), polyclonal mouse anti- ${ }^{6}$-methylguanine-DNA methyltransferase (MGMT; cat. no. ZM-0461), citrate buffer $(0.01 \mathrm{M}$; $\mathrm{pH}$ 6.0), modified phosphate-buffered saline (MPBS; $0.01 \mathrm{M}$; pH 7.2-7.6), and Tris-buffered saline containing Tween-20 (TBS-T; 0.01M, pH 7.2-7.6) were obtained from Beijing Zhongshan Golden Bridge Biotechnology Co., Ltd. (Beijing, China). Secondary antibodies, including Alexa fluor 488-conjugated goat anti-rabbit immunoglobulin (Ig)G (cat. no. A-11008) and Alexa fluor 555-conjugated donkey anti-mouse IgG (cat. no. A-31570), were purchased from Invitrogen Life Technologies, Inc. (Carlsbad, CA, USA). Reagents used for western blot analyses, including radioimmunoprecipitation assay (RIPA) lysis buffer, the bicinchoninic acid (BCA) protein assay kit, the $\beta$-actin primary antibody (cat. no. AA128), horseradish peroxidase (HRP)-conjugated goat anti-rabbit (cat. no. A0208) anti-mouse (cat. no. A0216) IgG secondary antibody, phenylmethyl sulfonylfluoride (PMSF), DAPI and the enhanced chemiluminescence (ECL) kit were purchased from the Beyotime Institute of Biotechnology (Shanghai, China). The HiFi-Moloney murine leukemia virus (MMLV) first-strand cDNA synthesis kit, UltraSYBR Mixture (with Rox) and DNase I were obtained from Beijing CW Biotech Co., Ltd. (Beijing, China).

Sample preparation. Brain tissues obtained from subjects were divided into two portions. One portion was prepared for histological examination, immunohistochemistry and immunofluorescence. The other portion was prepared for western blot analysis and reverse-transcription quantitative polymerase chain reaction (RT-qPCR). Briefly, the former set was fixed in $10 \%$ formalin for $15 \mathrm{~h}$ at room temperature, dehydrated using a graded ethanol series (75\% ethanol for $2 \mathrm{~h}, 85 \%$ ethanol for $2 \mathrm{~h}, 95 \%$ ethanol for $1 \mathrm{~h}$ and $100 \%$ ethanol for $1 \mathrm{~h}$ ), cleared in xylene, embedded in paraffin and stored at $4^{\circ} \mathrm{C}$ until use. The latter set was stored at $-80^{\circ} \mathrm{C}$ until use.

Histological examination. For histological studies, the paraffin-embedded samples were de-paraffinized in xylene and re-hydrated in a graded ethanol series $(100 \%$ ethanol for $5 \mathrm{~min}, 100 \%$ ethanol for $5 \mathrm{~min}, 95 \%$ ethanol for $5 \mathrm{~min}$, 95\% ethanol for $5 \mathrm{~min}, 85 \%$ ethanol for $3 \mathrm{~min}, 75 \%$ ethanol for $2 \mathrm{~min}$, and distilled water for $1 \mathrm{~min}$ ). Next, the samples were stained with hematoxylin solution (Beijing Dingguo Changsheng Biotechnology Co., Ltd., Beijing, China) for $5 \mathrm{~min}$, differentiated by soaking in $1 \%$ hydrochloric acid alcohol (pH 5.0; Beijing CW Biotech Co., Ltd.) for $15 \mathrm{sec}$ and washed under running tap water for $2 \mathrm{~min}$. After rinsing in $75 \%$ ethanol for $2 \mathrm{~min}$ and $85 \%$ ethanol for $3 \mathrm{~min}$, the sections were then counterstained with $0.5 \%$ eosin solution (Beijing Dingguo Changsheng Biotechnology Co., Ltd.) for $1 \mathrm{~min}$. Samples were then de-hydrated using two changes of $95 \%$ ethanol and two changes of $100 \%$ ethanol, cleared in xylene and mounted with neutral balsam (Beijing Dingguo Changsheng Biotechnology Co., Ltd.).

Immunohistological and immunofluorescence analyses. The paraffin-embedded sections were de-paraffinized and re-hydrated as described above. Peroxidase activity was inactivated by incubating samples for $30 \mathrm{~min}$ in $0.3 \%$ hydrogen peroxide in methanol (Changsha Organic Reagents Factory, Changsha, China). Samples were then processed for antigen retrieval by heating in citrate buffer for $5 \mathrm{~min}$ at $100^{\circ} \mathrm{C}$. After blocking in 10\% goat serum (cat. no. SP-9001; reagent A; Beijing Zhongshan Golden Bridge Biotechnology Co., Ltd.) for $2 \mathrm{~h}$ at $37^{\circ} \mathrm{C}$, sections were immunostained with the following primary antibodies overnight at $4^{\circ} \mathrm{C}$ : ADA (1:500 dilution), ADK (1:50 dilution), GFAP (1:150 dilution), Ki67 (1:100 dilution), TP53 (1:70 dilution for ADK co-labeling and 1:100 dilution for ADA co-labeling) or MGMT (1:100 dilution). After washing three times with MPBS, sections were incubated with the appropriate HRP-conjugated secondary antibody (cat. no. SP-9001, reagent B) for $30 \mathrm{~min}$ at $37^{\circ} \mathrm{C}$. Antibodies were stained using the DAB reagent. Subsequently, all sections were counterstained with hematoxylin and visualized using light microscopy (BX-50; Olympus, Tokyo, Japan). Images were captured using a LEICA DM5000B microscope (Leica Microsystems GmbH, Wetzlar, Germany) at x400 magnification, and five photomicrographs were randomly selected from each section. The number of immunopositive cells was estimated by averaging the number of cells counted per area. 
Table I. Primary sequences used for quantitative polymerase chain reaction analysis.

Target

gene

ADA

$\beta$-actin
Sequence

Length of amplified

product, bp

Forward, 5'-GGCTAACTACTCGCTCAACA-3'

Reverse, 5'-CGCATTGATGTTCAGCCTTT-3'

Reverse, 5'-CTACAAGCATTACCATACCC-3'

Forward, 5'-CTCCATCCTGGCCTCGCTGT-3'

268

Reverse, 5'-GCTGTCACCTTCACCGTTCC-3'

Table II. Clinical characteristics of study subjects.

\begin{tabular}{|c|c|c|c|c|c|c|c|c|c|}
\hline \multirow[b]{2}{*}{ Group } & \multirow[b]{2}{*}{$\mathrm{n}$} & \multirow{2}{*}{$\begin{array}{l}\text { Age range } \\
\text { (years) }\end{array}$} & \multicolumn{2}{|c|}{ Gender (n) } & \multicolumn{4}{|c|}{ Tumor grade (n) } & \multirow{2}{*}{$\begin{array}{l}\text { Glioma-associated } \\
\text { epilepsy cases (n) }\end{array}$} \\
\hline & & & Male & Female & I & II & III & IV & \\
\hline Tumor tissues & 45 & $2-65$ & 30 & 15 & 4 & 18 & 17 & 6 & 16 \\
\hline Peritumoral tissues & 14 & $18-55$ & 5 & 9 & 1 & 8 & 4 & 1 & 8 \\
\hline Normal control & 8 & $27-46$ & 5 & 3 & - & - & - & - & 0 \\
\hline
\end{tabular}

For immunofluorescence analyses, goat serum-blocked sections were incubated overnight at $4{ }^{\circ} \mathrm{C}$ with primary antibodies for ADA (1:30 dilution), ADK (1:20 dilution), p53 (1:70 or 1:100, dilution) alone or in combination. After washing in MPBS, sections were incubated for $3 \mathrm{~h}$ at room temperature with Alexa fluor-conjugated secondary antibodies. Nuclei were counterstained with DAPI. Images were randomly selected from various fields and captured using a LEICA DM5000B microscope under x200/400 magnification.

Western blot analysis. Total protein was extracted from cells using RIPA lysis buffer containing $1 \mathrm{mM}$ phenylmethylsulfonyl fluoride. The protein concentration in each sample was measured using a BCA protein assay kit according to manufacturer's instructions. Equal amounts of protein $(80 \mu \mathrm{g})$ were separated by $10 \%$ SDS-PAGE and transferred onto polyvinylidine fluoride membranes. Membranes were blocked with TBS containing $0.1 \%$ Tween-20 with $5 \%$ (w/v) non-fat dry milk, followed by overnight incubation at $4{ }^{\circ} \mathrm{C}$ with the following primary antibodies: ADA (1:600 dilution) plus $\beta$-actin (1:1,000 dilution) or ADK (1:1,000 dilution) plus $\beta$-actin (1:1,000 dilution). After washing three times with TBS-T, membranes were incubated for $2 \mathrm{~h}$ at room temperature with HRP-labeled anti-rabbit (1:1,000 dilution) and anti-mouse (1:1,000 dilution) secondary antibodies. After washing in TBS-T, immunoreactive bands were visualized using the ECL kit. To quantify protein levels, the expression bands of target proteins were analyzed using a ChemiDoc XRS gel imaging system (Image Lab 4.1; Bio-Rad Laboratories, Inc., Hercules, CA, USA). Densitometric values were used for statistical analyses. The housekeeping protein $\beta$-actin was used as an internal control.

$R T-q P C R$. Total RNA was extracted from brain tissues using an RNA extraction kit (Beijing CW Biotech Co., Ltd.) following the manufacturer's instructions. Total RNA $(1 \mu \mathrm{g})$ was reverse-transcribed using the HiFi-MMLV first-strand cDNA synthesis kit, and qPCR was performed using an ABI 7500 sequence detection system (Applied Biosystems Inc.). PCR amplification was performed using UltraSYBR Mixture (with Rox) with the specific primers listed in Table I (obtained from Beijing CW Biotech Co., Ltd.). PCR reactions were performed in duplicate at $95^{\circ} \mathrm{C}$ for $10 \mathrm{~min}$ followed by 40 cycles of $95^{\circ} \mathrm{C}$ for $15 \mathrm{sec}$ and $60^{\circ} \mathrm{C}$ for $60 \mathrm{sec}$, with a final extension step at $72^{\circ} \mathrm{C}$ for $5 \mathrm{~min}$. Each experimental condition was repeated in triplicate. The relative expression from amplified RNA samples was calculated using the $2^{-\Delta \Delta C T}$ method (17).

Statistical analysis. Values are expressed as the mean \pm standard deviation. All data were analyzed using SPSS 16.0 software (SPSS, Inc., Chicago, IL, USA) and plotted using Microsoft Excel 2003 (Microsoft Inc., Redmont, WA, USA). Statistical significance was determined using non-parametric tests, one-way analysis of variance and Student's $t$ tests. $\mathrm{p}<0.05$ was considered to indicate a statistically significant difference.

\section{Results}

Histological characteristics of glioma of various grades. The clinical characteristics of patients with glioma are presented in Table II. Among the 45 patients included, 16 cases exhibited glioma-associated epilepsy. None of the control subjects had a history of or current epilepsy. According to the WHO tumor grading system (16), four cases were classified as Grade I gliomas, 18 cases as Grade II, 17 cases as Grade III and six cases were considered Grade IV (Table II). Representative histological images are shown in Fig. 1. Grade-I astrocytoma showed slightly increased cell density and nuclear division was rarely 


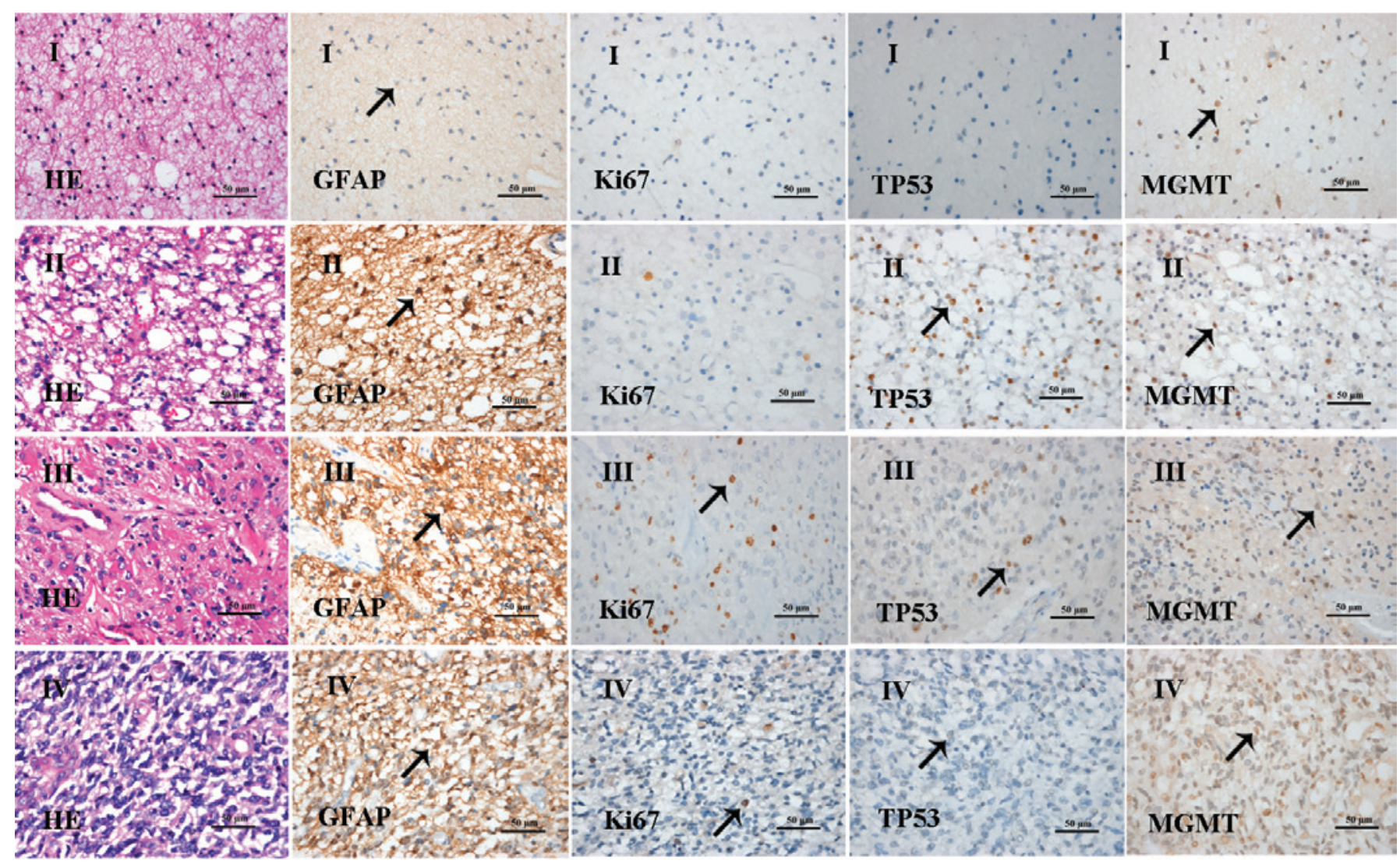

Figure 1. Histological examination of gliomas derived from patients. Samples from patients with Grade I, II, III or IV disease were stained with HE or with specific antibodies against GFAP, Ki67, p53 and MGMT (magnification, x400). Immunopositive cells are indicated by arrows. HE, hematoxylin and eosin; GFAP, glial fibrillary acidic protein; MGMT, $\mathrm{O}^{6}$-methylguanine-DNA methyltransferase.
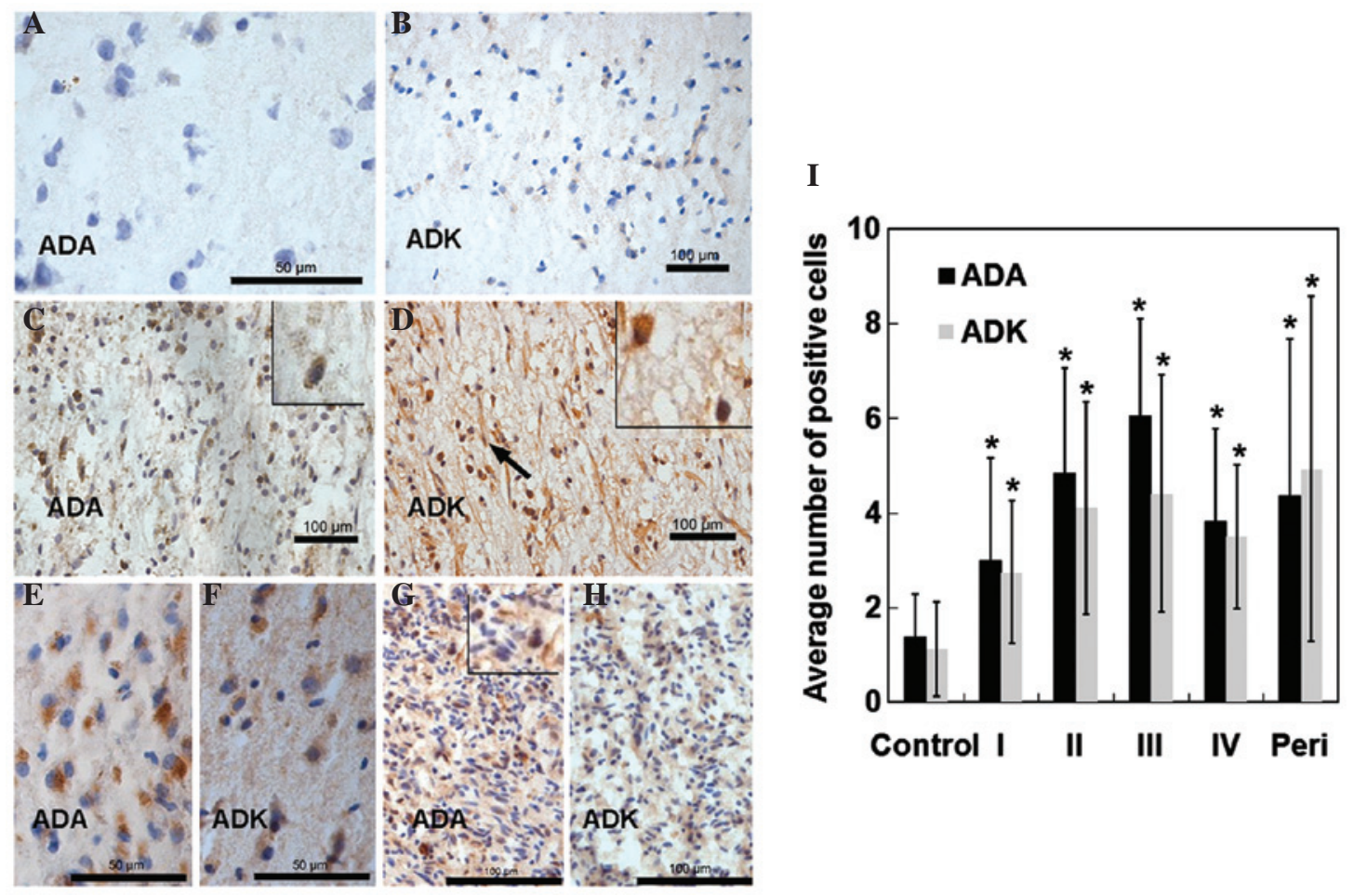

Figure 2. ADA and ADK expression in tissues from patients with various grades of glioma or from non-glioma and non-epileptic patients (normal controls). Normal brain tissues were stained with either (A) anti-ADA or (B) anti-ADK antibodies. Tumor tissues derived from patients with (C and D) Grade II, (E and F) Grade III or (G and H) Grade IV gliomas were stained with (C, E and G) anti-ADA or (D, F and H) anti-ADK antibodies (scale bars: $50 \mu \mathrm{m}$ for A-F; $100 \mu \mathrm{m}$ for $\mathrm{G}$ and $\mathrm{H}$ for. Magnification: A, B, C, D, E, and F, and magnified boxes in C, D, and G x400; G and H, x200). (I) Average number of immunopositive cells per field of view. Values are expressed as the mean \pm standard deviation. " $\mathrm{p}<0.05$ vs. control. Peri, peritumoral tissues derived from patients with glioma; ADA, adenosine deaminase; ADK, adenosine kinase. 
A

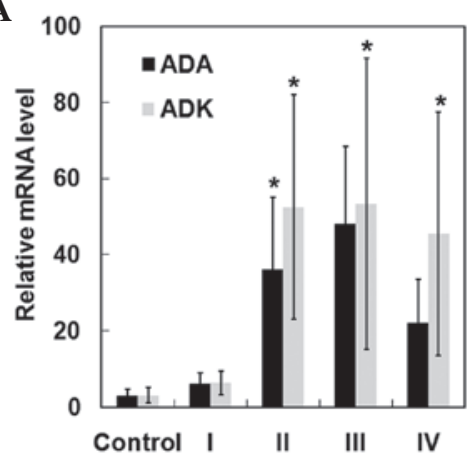

C

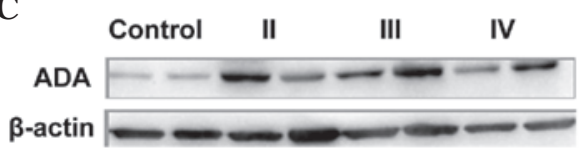

B
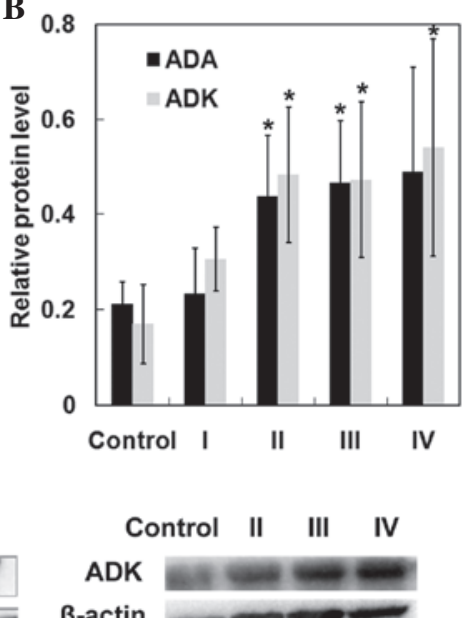

Figure 3. ADA and ADK mRNA and protein expression in brain tissues derived from patients with different glioma disease grades or from non-glioma and non-epileptic patients (normal controls). Samples were obtained from control subjects ( $\mathrm{n}=8$ ) or patients with Grade I ( $\mathrm{n}=4)$, Grade II ( $\mathrm{n}=18$ ), Grade III ( $\mathrm{n}=17$ ), or Grade IV ( $\mathrm{n}=6)$ glimoas. (A) Relative ADA and ADK mRNA expression was examined using reverse-transcription quantitative polymerase chain reaction analysis. (B) Protein expression of ADA and ADK was examined by western blot analyses. (C) Representative western blots. Values are expressed as the mean \pm standard deviation. ${ }^{*}$ p $<0.05$ vs. control. ADA, adenosine deaminase; ADK, adenosine kinase.

observed. Tumor cells were predominantly GFAP-positive and MGMT-positive, with a number of cells positive for Ki67 staining. In Grade-II oligodendroglioma, a moderate increase in tumor-cell density was noted, with few nuclear pleomorphisms and rare nuclear division. Tumor cells were largely GFAP-positive, TP53-positive and MGMT-positive. In addition, a number of cells were positive for Ki67 staining. Grade-III anaplastic astrocytoma exhibited local or diffuse cell proliferation, anaplasia and excessive nuclear division. Tumor cells were GFAP-positive, Ki67-positive and MGMT-positive, and a number of cells were positive for TP53. Grade-IV glioblastoma showed enhanced cell density, atypical shapes, poor differentiation, marked nuclear pleomorphisms, nuclear division, microvessel proliferation, thrombosis and tissue necrosis. Tumor cells were predominantly GFAP-positive, Ki67-positive and MGMT-positive.

$A D A$ and $A D K$ are overexpressed in glioma tissues from patients with various disease grades. The present study next evaluated the distribution of ADA and ADK in tumor tissues obtained from patients with various glioma grades. As shown in Fig. 2A and B, ADA and ADK were expressed at low levels in control brain tissues. Increased numbers of ADA-positive and ADK-positive cells were observed in patients with Grade I-IV gliomas compared to those in the normal tissues (Fig. 2C-H). ADA was primarily distributed throughout the cytoplasm of tumor cells, whereas ADK was expressed in the cytoplasm as well as the nuclei. Statistical analyses showed that the number of ADA-positive and ADK-positive cells was significantly upregulated in tumor tissues from patients with different glioma grades compared to that in brain tissues from control cases $(\mathrm{p}<0.05)$ (Fig. 2I). In addition, RT-qPCR and western blot analyses further confirmed significant upregulation of ADA and ADK in patients with Grade II-IV gliomas (Fig. 3). These results demonstrated that ADA and ADK expression was upregulated in patients with Grade II-IV gliomas.
$A D A$ and $A D K$ are overexpressed in peritumoral tissues. Similarly to that in glioma tissues, ADA and ADK expression in peritumoral tissues derived from patients with glioma $(n=14)$ was markedly elevated compared with that in control tissues $(\mathrm{p}<0.05)$ (Fig. 2B). Therefore, immunofluorescence assays were performed to investigate tumor invasion in peritumoral tissues. As revealed in Fig. 4, TP53-positive tumor cells were observed in peritumoral tissues derived from glioma patients. Furthermore, ADA and TP53 were co-expressed in numerous cells, with ADA located in the cytoplasm and TP53 located in the nuclei. Similarly, ADK and TP53 co-localization was observed, with TP53 located in the nuclei and ADK located in the cytoplasm and nuclei. These results confirmed tumor invasion in peritumoral tissues.

$A D A$ and $A D K$ expression is increased in peritumoral tissues from glioma patients with epilepsy vs. non-epilepsy patients. Finally, the present study compared ADA and ADK expression between glioma patients with and without epilepsy. As shown in Fig. 5A, the number of ADA-positive or ADK-positive cells was markedly increased in peritumoral tissues derived from glioma patients associated with epilepsy compared to that in patients without epilepsy. Statistical analyses revealed no significant differences in the number of ADA-positive and ADK-positive cells in tumor tissues derived from glioma patients with or without epilepsy ( $p>0.05$ ) (Fig. 5B). However, the levels of ADA and ADK tended to be higher in peritumoral tissues from patients associated with epilepsy compared to those in glioma patients without epilepsy $(\mathrm{p}<0.05)$. Furthermore, western blot analysis confirmed upregulated ADA and ADK expression in peritumoral tissues derived from patients with epilepsy (Fig. 5C and D).

\section{Discussion}

The present study assessed ADA and ADK expression in tumor tissues and peritumoral tissues derived from glioma 


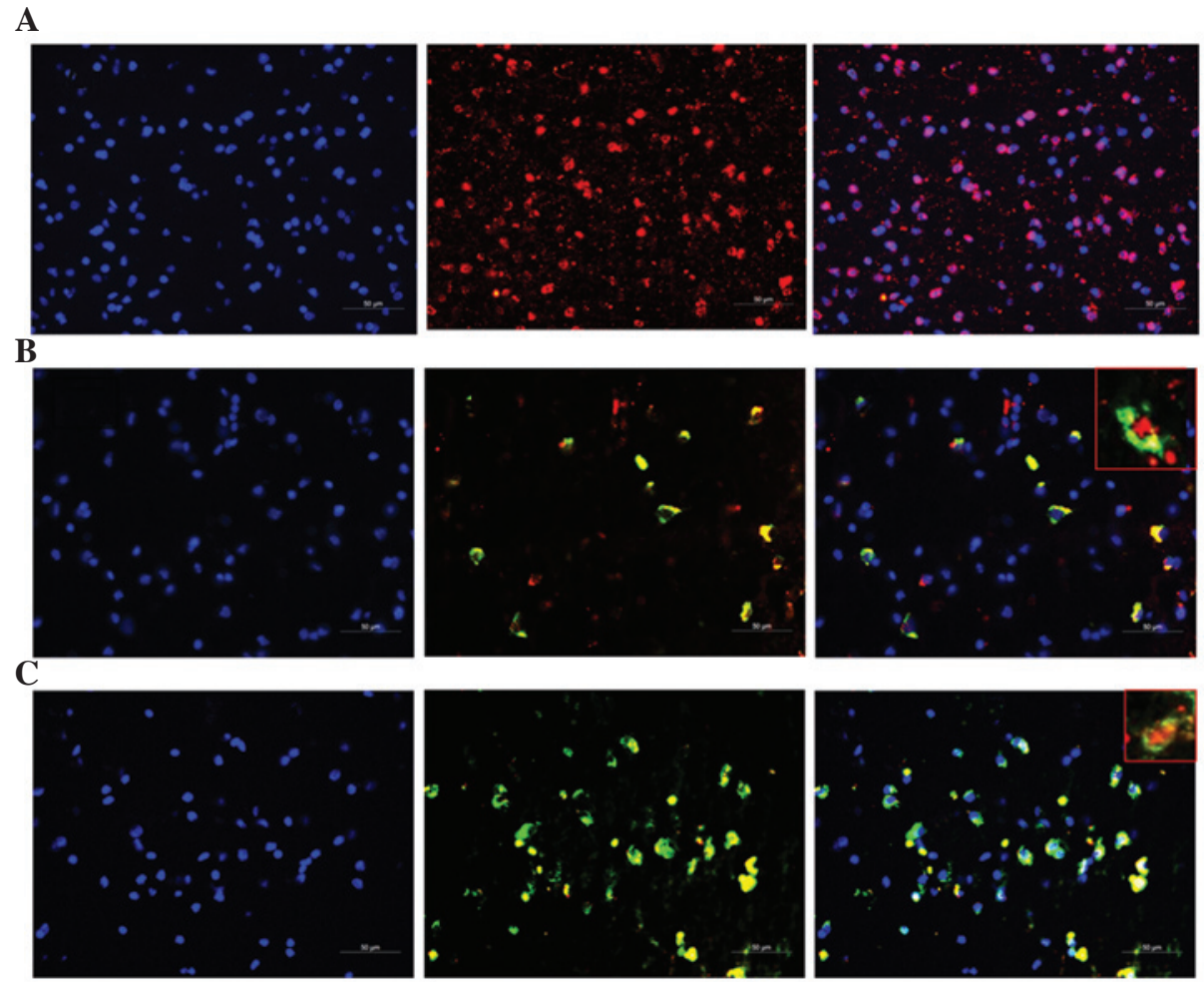

Figure 4. Immunostaining of peritumoral tissues with anti-p53, anti-ADA, and anti-ADK antibodies (magnification, $\mathrm{x} 200$; magnified boxes, $\mathrm{x} 400$ ). Peritumoral tissues derived from 14 patients with glioma were immunostained with the anti-p53 antibody alone (red) or co-stained with anti-p53 (red) and anti-ADA or anti-ADK (green) antibodies. Nuclei were counterstained using DAPI (blue). Representative immunostaining images for (A) p53 alone (red), (B) ADA (green) and p53 (red), and (C) ADK (green) and p53 (red). Left-hand column, cell nuclei; middle column, immunostaining; right-hand column, merged image, containing magnified window (scale bars, $50 \mu \mathrm{m}$ ). ADA, adenosine deaminase; ADK, adenosine kinase.

A

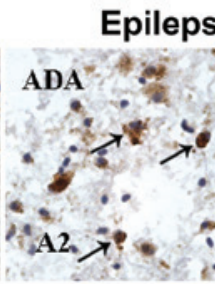

sy

Epilepsy

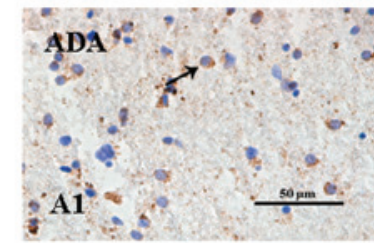

B

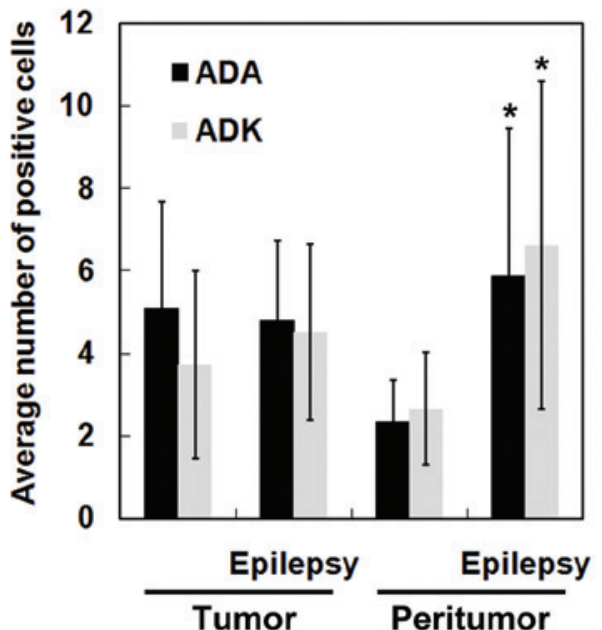

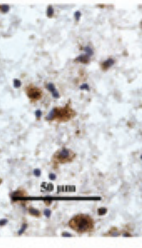

C

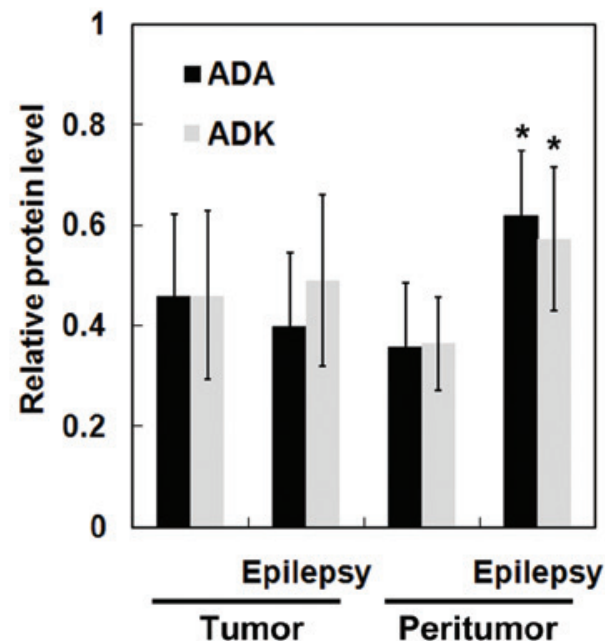

D

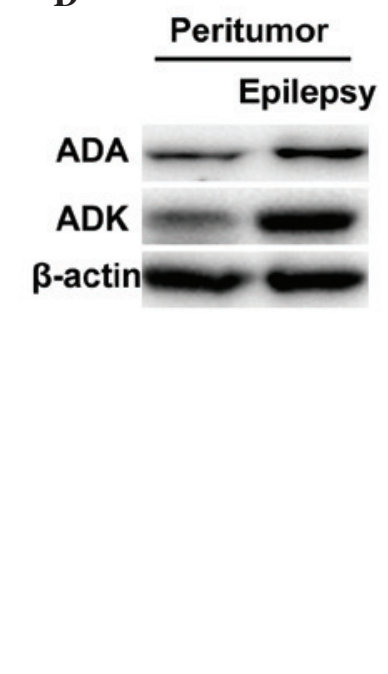

Figure 5. ADA and ADK expression in glioma patients with or without epilepsy. (A) Peritumoral tissues were immunostained with anti-ADA or anti-ADK antibodies (magnification A1, A2, A3, and A4, x400). Boxed images are magnified from the original images. Positive staining is indicated by arrows. (B) Number of immunopositive cells per area. (C) Western blot analysis of ADA and ADK protein expression. (D) Representative western blot. Values are expressed as the mean \pm standard deviation. Tumor tissues derived from patients without epilepsy $(n=29)$; tumor tissues derived from patients associated with epilepsy $(n=16)$; peritumoral tissues derived from patients without epilepsy $(n=6)$; peritumoral tissues derived from patients with epilepsy ( $n=8)$. " $p<0.05$ vs. peritumoral tissues derived from patients without epilepsy. ADA, adenosine deaminase; ADK, adenosine kinase. 
patients and compared their expression levels with those in brain tissues from normal control subjects. In addition, ADA and ADK levels were compared between glioma patients with and without epilepsy.

Adenosine is an endogenous mediator of brain function $(10,11)$. Metabolic disorders of purine metabolism, including adenosine metabolism, affect the nervous system (18) and are linked to glioma in humans (19). Castillo et al (20) reported that moderate hypoxia modulates adenosine receptors in C6 glioma cells, which suggests that the activation of endogenous adenosine receptors occurs during hypoxia.

ADA and ADK are capable of regulating adenosine levels and may thus contribute to abnormal adenosine metabolism-induced diseases. ADA has a critical role in purine metabolism by catalyzing the deamination of adenosine and deoxyadenosine. In a study on C6 glioma cells, ADA was demonstrated to eliminate adenosine-mediated growth inhibition of cells (21). Furthermore, ADK, another enzyme associated with adenosine metabolism, has also been shown to be associated with human gliomas. ADK expression was found to be significantly increased in human astrocytic tumors and peritumoral tissues compared to levels detected in normal white matter and normal cortices (14).

In the present study, increased ADA and ADK expression was noted in tumor tissues and peritumoral tissues compared to that in normal control tissues, and these findings were consistent with those from a previous study (14). Peritumoral tissues containing invading tumor cells were found to be immunopositive for TP53. Furthermore, ADA and ADK levels were found to be upregulated particularly in patients with Grade II-IV gliomas. These results demonstrated that ADA and ADK levels may be associated with glioma pathogenesis and progression. However, the underlying mechanism of the roles of ADK and ADA in glioma requires further study.

Epilepsy is a frequent clinical manifestation of glioma. Considering the anti-convulsive role of endogenous adenosine, modulation of the adenosinergic system has been suggested to be a potential target for the treatment of epilepsy (22). A previous study demonstrated significantly elevated ADK expression in peritumoral infiltrated tissues from patients with epilepsy compared to that in patients without epilepsy (14). In addition, ADA has been shown to be involved in controlling seizure development in zebrafish, and anti-epileptic drugs administered during these acute seizure episodes in the zebrafish prevented changes in adenosine desamination (15). In accordance with these observations, the present study found that ADA and ADK were upregulated in peritumoral tissues from patients with epilepsy, as compared to those in glioma patients without epilepsy. However, no significant differences were observed in the number of ADA- or ADK-positive cells in tumor tissues from epileptic and non-epileptic glioma patients. It is possible that increased ADA and ADK expression may induce excessive adenosine degradation, resulting in a reduced inhibitory role of adenosine and subsequent epilepsy attacks in glioma patients $(23,24)$.

Adenosine augmentation therapies (AATs), which upregulate the adenosine concentration in the brain and thereby help to control the occurrence of seizures, have been demonstrated to be effective in managing epilepsy (25). ADA and ADK have critical roles in the metabolism of adenosine as well as the development of epilepsy. Accumulating evidence suggested that ADK may represent a therapeutic target for AATs. For example, upregulation of the local adenosine concentration by transplantation of encapsulated adenosine-releasing cells, including kidney fibroblasts (26), mouse myoblasts (27) and mouse embryonic stem cells $(28,29)$, into the lateral ventricle acts against brain damage and epilepsy by disrupting ADK gene expression. Using zebrafish as a model organism, ADA was found to be involved in the development of epilepsy (15). In analogy with these findings, the present study also indicated that ADA was involved in the development and progression of human epilepsy. Hence, ADA may be a novel target for AATs. Administration of ADA or ADK inhibitors may also be a feasible approach for anti-epilepsy therapies. To the best of our knowledge, the present study was the first to report ADA upregulation in glioma-associated epilepsy.

In conclusion, the present study demonstrated that ADA and ADK levels were upregulated in patients with gliomas with and without epilepsy. Furthermore, ADA and ADK expression was upregulated in the peritumor tissues of patients with glioma-associated epilepsy as compared with those in non-epileptic patients. These findings suggested an essential role for adenosine metabolism in regulating glioma pathogenesis and progression as well as in glioma-associated epilepsy.

\section{Acknowledgements}

The present study was supported by a grant from the Department of Science and Technology of Hunan Province, P.R. China (no. 2013FJ4367).

\section{References}

1. Pace A, Bove L, Innocenti P, Pietrangeli A, Carapella CM, Oppido P, Raus L, Occhipinti E and Jandolo B: Epilepsy and gliomas: Incidence and treatment in 119 patients. J Exp Clin Cancer Res 17: 479-482, 1998.

2. Behin A, Hoang-Xuan K, Carpentier AF and Delattre JY: Primary brain tumours in adults. Lancet 361: 323-331, 2003.

3. Pallud J, Audureau E, Blonski M, Sanai N, Bauchet L, Fontaine D, Mandonnet E, Dezamis E, Psimaras D, Guyotat J, et al: Epileptic seizures in diffuse low-grade gliomas in adults. Brain 137: 449-462, 2014.

4. Berntsson SG, Malmer B, Bondy ML, Qu M and Smits A: Tumor-associated epilepsy and glioma: Are there common genetic pathways? Acta Oncol 48: 955-963, 2009.

5. Lynam LM, Lyons MK, Drazkowski JF, Sirven JI, Noe KH, Zimmerman RS and Wilkens JA: Frequency of seizures in patients with newly diagnosed brain tumors: A retrospective review. Clin Neurol Neurosurg 109: 634-638, 2007.

6. Stieber VW: Low-grade gliomas. Curr Treat Options Oncol 2: 495-506, 2001

7. Lee MC, Kim GM, Woo YJ, Kim MK, Kim JH, Nam SC, Suh JJ, Chung WK, Lee JS, Kim HI, et al: Pathogenic significance of neuronal migration disorders in temporal lobe epilepsy. Hum Pathol 32: 643-648, 2001.

8. Aronica E, Gorter JA, Jansen GH, Leenstra S, Yankaya B and Troost D: Expression of connexin 43 and connexin 32 gap-junction proteins in epilepsy-associated brain tumors and in the perilesional epileptic cortex. Acta Neuropathol 101: 449-459, 2001.

9. Yuen TI, Morokoff AP, Bjorksten A, D'Abaco G, Paradiso L, Finch S, Wong D, Reid CA, Powell KL, Drummond KJ, et al: Glutamate is associated with a higher risk of seizures in patients with gliomas. Neurology 79: 883-889, 2012.

10. Boison D: Role of adenosine in status epilepticus: A potential new target? Epilepsia 54 (Suppl 6): 20-22, 2013. 
11. Boison D: Adenosine and seizure termination: Endogenous mechanisms. Epilepsy Curr 13: 35-37, 2013.

12. Hargus NJ, Jennings C, Perez-Reyes E, Bertram EH and Patel MK: Enhanced actions of adenosine in medial entorhinal cortex layer II stellate neurons in temporal lobe epilepsy are mediated via A(1)-receptor activation. Epilepsia 53: 168-176, 2012.

13. Boison D: Adenosine kinase: Exploitation for therapeutic gain. Pharmacol Rev 65: 906-943, 2013.

14. de Groot M, Iyer A, Zurolo E, Anink J, Heimans JJ, Boison D, Reijneveld JC and Aronica E: Overexpression of ADK in human astrocytic tumors and peritumoral tissue is related to tumor-associated epilepsy. Epilepsia 53: 58-66, 2012.

15. Siebel AM, Piato AL, Schaefer IC, Nery LR, Bogo MR and Bonan CD: Antiepileptic drugs prevent changes in adenosine deamination during acute seizure episodes in adult zebrafish Pharmacol Biochem Behav 104: 20-26, 2013.

16. Louis DN, Ohgaki H, Wiestler OD, Cavenee WK, Burger PC, Jouvet A, Scheithauer BW and Kleihues P: The 2007 WHO classification of tumours of the central nervous system. Acta Neuropathol 114: 97-109, 2007.

17. Livak KJ and Schmittgen TD: Analysis of relative gene expression data using real-time quantitative PCR and the 2(-Delta Delta C(T)) Method. Methods 25: 402-408, 2001.

18. Jinnah HA, Sabina RL and Van Den Berghe G: Metabolic disorders of purine metabolism affecting the nervous system. Handb Clin Neurol 113: 1827-1836, 2013.

19. Bardot V, Dutrillaux AM, Delattre JY, Vega F, Poisson M, Dutrillaux B and Luccioni C: Purine and pyrimidine metabolism in human gliomas: Relation to chromosomal aberrations. $\mathrm{Br} J$ Cancer 70: 212-218, 1994.

20. Castillo CA, León D, Ruiz MA, Albasanz JL and Martín M: Modulation of adenosine $\mathrm{A} 1$ and A2A receptors in C6 glioma cells during hypoxia: Involvement of endogenous adenosine. J Neurochem 105: 2315-2329, 2008.
21. Ohkubo S, Nagata $K$ and Nakahata N: Adenosine uptake-dependent C6 cell growth inhibition. Eur J Pharmacol 577: 35-43, 2007.

22. Swiader MJ, Kotowski J and Łuszczki JJ: Modulation of adenosinergic system and its application for the treatment of epilepsy. Pharmacol Rep 66: 335-342, 2014.

23. Pence S, Erkutlu I, Kurtul N, Bosnak M, Alptekin M and Tan U: Antiepileptogenic effects of glutathione against increased brain ADA in PTZ-induced epilepsy. Int J Neurosci119: 616-629, 2009.

24. Shen HY, Sun H, Hanthorn MM, Zhi Z, Lan JQ, Poulsen D J, Wang RK and Boison D: Overexpression of adenosine kinase in cortical astrocytes and focal neocortical epilepsy in mice. J Neurosurg 120: 628-638, 2014.

25. Boison D: Adenosine augmentation therapies (AATs) for epilepsy: Prospect of cell and gene therapies. Epilepsy Res 85: 131-141, 2009.

26. Huber A, Padrun V, Déglon N, Aebischer P, Möhler H and Boison D: Grafts of adenosine-releasing cells suppress seizures in kindling epilepsy. Proc Natl Acad Sci USA 98: 7611-7616, 2001.

27. Güttinger M, Padrun V, Pralong WF and Boison D: Seizure suppression and lack of adenosine A1 receptor desensitization after focal long-term delivery of adenosine by encapsulated myoblasts. Exp Neurol 193: 53-64, 2005.

28. Güttinger M, Fedele D, Koch P, Padrun V, Pralong WF, Brüstle O and Boison D: Suppression of kindled seizures by paracrine adenosine release from stem cell-derived brain implants. Epilepsia 46: 1162-1169, 2005.

29. Fedele DE, Koch P, Scheurer L, Simpson EM, Möhler H, Brüstle $\mathrm{O}$ and Boison D: Engineering embryonic stem cell derived glia for adenosine delivery. Neurosci Lett 370: 160-165, 2004. 